\title{
PARTIALLY ORDERED LINEAR ALGEBRAS WITH MULTIPLICATIVE DIAGONAL MAP
}

\author{
BY \\ TAEN-YU DAI AND RALPH DEMARR
}

\begin{abstract}
The diagonal of the product of two triangular matrices is the product of the diagonals of each matrix. This idea is used to characterize partially ordered linear algebras which have order properties similar to an algebra of real triangular matrices.
\end{abstract}

1. Introduction and basic definitions. This paper is motivated by the work of Kadison and Singer [6] on triangular operator algebras, but instead of dealing with a normed linear algebra of operators, we consider an abstract partially ordered linear algebra (pola). It should be pointed out that the algebra of all norm bounded operators on a real Banach space can always be regarded as a pola [3]. Although the basic concept is the same as in [6], our approach is somewhat different and centers on the fact that in an algebra of upper triangular matrices the diagonal of the product of two matrices is equal to the product of the diagonals (see §4). The examples in $\S 2$ will illustrate this difference.

The basic references on partially ordered linear spaces and algebras are [1], [8], [9] , [10]. The basic references on operator algebras with a "diagonal" and on triangular operator algebras are [4], [5], [6], [7]. In this paper the term "operator" always refers to a linear operator.

The basic definitions needed in this paper will now be given. The basic reference is Dai [2], but the reader should note that the term "polac" used there has the same meaning as "dsc-pola" used in this paper. A partially ordered linear algebra (pola; denoted by $A$ ) is a real linear associative algebra which is partially ordered so that it is a directed partially ordered linear space and $0 \leqslant x y$ whenever $x, y \in A, 0 \leqslant x, 0 \leqslant y$. We also assume that $A$ has a multiplicative identity $1 \geqslant 0$. $A$ Dedekind $\sigma$-complete pola (dsc-pola) $A$ is one having the property: If $x_{n} \in A, 0 \leqslant \cdots \leqslant x_{2} \leqslant x_{1}$, then $\inf \left\{x_{n}\right\}$ exists. Order convergence is defined as usual. A dsc-pola $A$ has the Archimedean property: if $x, y \in A$ and $n x \leqslant y$ for every positive integer $n$, then $x \leqslant 0$.

The fact that $A$ is directed plays an important role. This means that if $x$

Received by the editors September 24, 1975.

AMS (MOS) subject classifications (1970). Primary 06A70; Secondary 15A45, 47B55.

Key words and phrases. Dedekind $\sigma$-complete partially ordered linear algebra, diagonal, triangular matrices, matrix inequalities, triangular operators. 
$\in A$, then there exists $y \in A$ such that $0 \leqslant y$ and $x \leqslant y$. The reader can easily verify that this is equivalent to the following assumption: If $x \in A$, then there exist elements $y, z \in A$ such that $0 \leqslant y, 0 \leqslant z$ and $x=y-z=(1+y)-$ $(1+z)$.

We define addition and multiplication of subsets as usual. Thus, if $S_{1}$ and $S_{2}$ are any two nonempty subsets of $A$ and $\beta$ is a real number, we define $\beta S_{1} \pm$ $S_{2}=\left\{\beta x_{1} \pm x_{2}: x_{1} \in S_{1}\right.$ and $\left.x_{2} \in S_{2}\right\}$ and $S_{1} S_{2}=\left\{x_{1} x_{2}: x_{1} \in S_{1}\right.$ and $x_{2} \in$ $\left.S_{2}\right\}$.

We now define the diagonal part of a dsc-pola. It is important to point out that this is an "order concept" and does not necessarily relate to the concept of a diagonal as discussed by Kadison and Singer. We first define $I=\{y: y \geqslant 1$ and $\left.y^{-1} \geqslant 0\right\}$ and then define $A_{1}=I-I$. We call $A_{1}$ the diagonal (or functional) part of $A$. The elements of $A_{1}$ behave like diagonal matrices or realvalued functions. We will summarize the basic properties of $A_{1}$ in $\S 3$.

We next define a diagonal projection (DP) map $\Delta: A \rightarrow A_{1}$ as a linear map which has the following two properties: (1) $\Delta(1)=1$; (2) if $x \geqslant 0$, then $0 \leqslant \Delta(x) \leqslant x$. Since $\Delta$ is linear, it follows from (2) that $\Delta$ is isotone. This map was considered by Dai; see p. 671 of [2], but note that Dai uses $d(\cdot)$ instead of $\Delta(\cdot)$. He shows that if $A$ is a lattice, then a DP map always exists, but Example 4 in $\S 2$ shows that in some cases a DP map may not exist. In $\S 3$ it will be shown that if $\Delta$ exists, then it is unique. In $\S 4$ we discuss various natural properties which imply that $\Delta$ is multiplicative. The reader should note that a diagonal projection map is related to the so-called "diagonal process" defined by Kadison and Singer; see p. 387 of [5] or p. 109 of [4].

2. Examples. The following examples are given to illustrate the basic concepts involved. In particular, they illustrate the difference between our approach and that of Kadison and Singer. In order to keep the notation simple, we make the following convention: In each example we give a real linear algebra $A$ and then define a subset $K \subset A$ which acts as the "positive cone." As usual, if $x, y$ $\in A$, then $x \leqslant y$ means that $y-x \in K$. The reader may fill in the necessary details.

The first four examples are quite simple and are intended to show that the same real linear algebra can be partially ordered in various ways to give quite different results.

EXAMPLE 1. The real linear algebra $A$ is the set of quadruples $x=$ $\left(\alpha_{1}, \alpha_{2}, \alpha_{3}, \alpha_{4}\right)$ of real numbers, where we add and multiply componentwise. Multiplication by a real scalar is defined in the same way. By definition $x \in K$ if and only if $0 \leqslant \alpha_{i}$ for $i=1,2,3,4$. Thus, $A$ is a dsc-pola and $A=A_{1}$. Hence, we may define $\Delta(x)=x$ for all $x \in A$. 
Example 2. The real linear algebra $A$ is the same as in Example 1. By definition $x \in K$ if and only if $0 \leqslant \alpha_{1} \leqslant \alpha_{2} \leqslant \alpha_{3} \leqslant \alpha_{4}$. Thus, $A$ is a dsc-pola and is also a lattice. It is easy to show that $A_{1}=\{\beta 1: \beta$ is a real number $\}$. We define $\Delta(x)=\alpha_{1} 1$.

Example 3. The real linear algebra $A$ is the same as in Example 1. By definition $x \in K$ if and only if $0 \leqslant \alpha_{1} \leqslant \alpha_{2} \leqslant \alpha_{4}$ and $\alpha_{1} \leqslant \alpha_{3} \leqslant \alpha_{4}$. Thus, $A$ is a dsc-pola (but not a lattice) and $A_{1}$ is the same as in Example 2. We define $\Delta(x)=\alpha_{1} 1$

ExAmple 4. The real linear algebra $A$ is the same as in Example 1. By definition $x \in K$ if and only if $0 \leqslant \alpha_{i} \leqslant \alpha_{4}$ for $i=1,2,3$. Thus, $A$ is a dscpola (but not a lattice) and $A_{1}$ is the same as in Example 2. However, in this example there is no diagonal projection map.

ExAMPLE 5. The real linear algebra $A$ is the algebra of matrices

$$
x=\left[\begin{array}{ll}
\alpha & \beta \\
\beta & \alpha
\end{array}\right],
$$

where $\alpha$ and $\beta$ are real numbers. By definition $x \in K$ if and only if $\alpha \geqslant 0$ and $\beta \geqslant 0$. Thus, $A$ is a dsc-pola and is also a lattice. It is easy to show that $A_{1}=$ $\{\lambda 1: \lambda$ is a real number $\}$. We define $\Delta(x)=\alpha 1$.

EXAmple 6. The real linear algebra $A$ is the algebra of infinite matrices $x=\left[\alpha_{i j}\right]$, where $i, j=1,2,3, \ldots$, which are in upper triangular form. This means that $\alpha_{i j}=0$ for $j<i$. Of course, the $\alpha_{i j}$ are real numbers. By definition $x \in K$ if and only if $\alpha_{i j} \geqslant 0$ for all $i, j$. Thus, $A$ is a dsc-pola and also a lattice. In this case $A_{1}$ is just the subalgebra of diagonal matrices. We define $\Delta(x)=$ $\left[\alpha_{i i} \delta_{i j}\right]$.

This example has a curious property which will be discussed later. If we define the diagonal matrix $a=\left[i \delta_{i j}\right]$, then for any $z \in A$, where $z \geqslant 0$ and $\Delta(z)$ $=0$, we have $z+a z \leqslant z a$.

EXAMPLE 7. This example is the same as Example 6 except that here we consider only row-finite matrices; that is, each row eventually consists only of zero entries.

EXAmple 8. This example is the same as Example 6 (or Example 7) except that here we require the sequence $\left\{\alpha_{i i}\right\}$ of diagonal entries to be a bounded sequence of real numbers.

EXAmple 9. This example is the same as Example 6 but here we consider infinite matrices in lower triangular form. This means that $\alpha_{i j}=0$ for $i<j$.

Although the above nine examples are quite elementary, they are sufficient for our purposes. More substantial examples may be found in [2]; see Example 5.6 on p. 679 and Example 5.7 on p. 680 . Examples relating to operators on a 
Hilbert space may be found in [6]. The reader will find an excellent discussion on lattices of operators in Chapter 4 of Schaefer [9].

3. General properties of a diagonal projection map. We first list those properties of the diagonal part $A_{1}$ (defined in $\$ 1$ ) that will be needed in this paper. Proofs of these properties can be found in [2].

Lemma 3.1. Basic Properties of the Diagonal Part. The diagonal part $A_{1}$ is nonempty and $1 \in A_{1}$. It is a commutative directed order-convex sub-dsc-pola of $A$ and is closed with respect to order convergence. Of particular importance are:

(1) if $a \in A_{1}$ and $a^{2}=0$, then $a=0$;

(2) if $a \in A_{1}$ and $0 \leqslant a^{n} \leqslant w(w \in A)$ for all $n=1,2, \ldots$, then $0 \leqslant$ $a \leqslant 1$

(3) if $b \in A_{1}$ and $b \geqslant 0$, then there exists $a \in A_{1}$ such that $0 \leqslant a \leqslant 1$ and $b=\Sigma_{k=1}^{\infty} a^{k}$;

(4) if $a, b \in A, a \geqslant 0, b \geqslant 1$ and $a b=1$, then $a, b \in A_{1}$;

(5) if $a \in A_{1}, b \in A$ and $a b=1$, then $b \in A_{1}$ and $b a=1$.

THEOREM 3.2. The basic properties of a diagonal projection map $\Delta$ are:

(1) if $a \in A_{1}$, then $\Delta(a)=a$;

(2) if $a \in A_{1}$ and $x \in A$, then $\Delta(a x)=\Delta(x a)=a \Delta(x)$;

(3) if $x, y \in A, x \geqslant 0$ and $y \geqslant 0$, then $0 \leqslant \Delta(x) \Delta(y) \leqslant \Delta(x y)$.

Proof. To prove part (1) we first note that if $0 \leqslant c \leqslant 1$, then we may put $b=1-c \geqslant 0$, so that $b-\Delta(b)+c-\Delta(c)=0$, where we use the fact that $\Delta(b+c)=\Delta(1)=1$. Since $b-\Delta(b) \geqslant 0$ and $c-\Delta(c) \geqslant 0$, we get $\Delta(c)=c$. Thus, if $0 \leqslant c \leqslant 1$, then $\Delta(c)=c$.

Now if we select any $d \in A_{1}, d \geqslant 0$, then there exists an element $c$ such that $0 \leqslant c \leqslant 1$ and $d=\Sigma_{k=1}^{\infty} c^{k}$. This is part (3) of Lemma 3.1. If we define the partial sums $s_{n}=\Sigma_{k=1}^{n} c^{k}$, then it can be shown by induction (using the result of the previous paragraph) that $\Delta\left(s_{n}\right)=s_{n}$ for all $n$. Now $0 \leqslant d-\Delta(d)$ $<d-\Delta\left(s_{n}\right)=d-s_{n}$ for all $n$. Since $\inf \left\{d-s_{n}\right\}=0$, we get $\Delta(d)=d$. Finally, since $A_{1}$ is directed, for any $a \in A_{1}$ we may find $d_{1}, d_{2} \in A_{1}$ such that $d_{1} \geqslant 0, d_{2} \geqslant 0$ and $a=d_{1}-d_{2}$. Thus, $\Delta(a)=\Delta\left(d_{1}\right)-\Delta\left(d_{2}\right)=d_{1}-d_{2}=a$.

To prove part (2) we proceed as we did above. Thus, if $0 \leqslant c \leqslant 1, b=$ $1-c, y \in A$ and $y \geqslant 0$, then $\Delta(y)=b \Delta(y)+c \Delta(y)=\Delta(b y)+\Delta(c y)$, so that $\Delta(b y)-b \Delta(y)+\Delta(c y)-c \Delta(y)_{0}=0$. Since $0 \leqslant b \Delta(y) \leqslant b y$ and $b \Delta(y) \in A_{1}$, we obtain $0 \leqslant b \Delta(y) \leqslant \Delta(b y)$. Thus, $\Delta(b y)-b \Delta(y) \geqslant 0$ and $\Delta(c y)-c \Delta(y) \geqslant$ 0 , so that $\Delta(c y)=c \Delta(y)$. To summarize: if $0 \leqslant c \leqslant 1, y \in A$ and $y \geqslant 0$, then $\Delta(c y)=c \Delta(y)$.

Now if we select any $d \in A_{1}, d \geqslant 1$, then $0 \leqslant d^{-1} \leqslant 1$. Hence, if $y \in A$ 
and $y \geqslant 0$, then $\Delta(y)=\Delta\left(d^{-1} d y\right)=d^{-1} \Delta(d y)$, so that $d \Delta(y)=\Delta(d y)$. Finally, we may use the fact that both $A$ and $A_{1}$ are directed to show that $\Delta(a x)=a \Delta(x)$ for any $a \in A_{1}$ and $x \in A$. Clearly we may repeat the above argument to show that $\Delta(x a)=\Delta(x) a$ for any $a \in A_{1}$ and $x \in A$. Since $A_{1}$ is a commutative sub-dsc-pola of $A$, it follows that $a \Delta(x)=\Delta(x) a$.

To prove part (3) we note that if $x \geqslant 0$ and $y \geqslant 0$, then $0 \leqslant \Delta(x) \leqslant x$ and $0 \leqslant \Delta(y) \leqslant y$, so that $0 \leqslant \Delta(x) \Delta(y) \leqslant x y$. Since $\Delta(x) \Delta(y) \in A_{1}$, we obtain the final result: $0 \leqslant \Delta(x) \Delta(y) \leqslant \Delta(x y)$.

The next theorem establishes the uniqueness of a diagonal projection map.

THEOREM 3.3. If $\Delta_{1}$ and $\Delta_{2}$ are both diagonal projection maps, then $\Delta_{1}(x)=\Delta_{2}(x)$ for all $x \in A$.

Proof. For $y \in A$ and $y \geqslant 0$ let us define $a_{1}=\Delta_{1}(y) \in A_{1}$ and $a_{2}=$ $\Delta_{2}(y) \in A_{1}$. Since $0 \leqslant a_{1} \leqslant y$, we get $a_{1}=\Delta_{2}\left(a_{1}\right) \leqslant \Delta_{2}(y)=a_{2}$. Similarly, we can show $a_{2} \leqslant a_{1}$, so that $\Delta_{1}(y)=\Delta_{2}(y)$. Since $A$ is directed, it follows that $\Delta_{1}(x)=\Delta_{2}(x)$ for all $x \in A$.

LEMMA 3.4. If $w, x \in A$ are such that $0 \leqslant x^{n} \leqslant w$ for all $n=1,2, \ldots$, then $0 \leqslant \Delta\left(x^{k}\right) \leqslant 1$ for all $k=1,2, \ldots$.

Proof. For each $k$ define $y=x^{k}$ and note that $0 \leqslant y^{n} \leqslant w$ for all $n$. Hence, $0 \leqslant \Delta(y)^{n} \leqslant \Delta\left(y^{n}\right) \leqslant \Delta(w) \leqslant w$ for all $n$ (we used part (3) of Theorem 3.2 here). From part (2) of Lemma 3.1 we obtain $0 \leqslant \Delta(y) \leqslant 1$. Hence, $0 \leqslant$ $\Delta\left(x^{k}\right) \leqslant 1$ for all $k=1,2, \ldots$.

We now introduce two important subsets of $A$ which will be used in $\S 4$. We define $N_{1}=\{z: z \geqslant 0$ and $\Delta(z)=0\}$ and $N_{2}=\left\{z: z \geqslant 0\right.$ and $\left.\Delta\left(z^{2}\right)=0\right\}$.

LEMMA 3.5. $N_{2} \subset N_{1}$.

Proof. If $z \in N_{2}$, then $0 \leqslant z$ and $0 \leqslant \Delta(z)^{2} \leqslant \Delta\left(z^{2}\right)=0$, so that $\Delta(z)^{2}=0$ (we used part (3) of Theorem 3.2 here). Since $\Delta(z) \in A_{1}$ and $\Delta(z)^{2}$ $=0$, we may use part (1) of Lemma 3.1 to obtain $\Delta(z)=0$. Hence, $z \in N_{1}$.

LEMMA 3.6. $N_{1}=N_{2}$ if and only if $N_{1} N_{1} \subset N_{1}$.

Proof. Suppose that $N_{1}=N_{2}$. Take elements $z_{1}, z_{2} \in N_{1}$ and put $z=z_{1}+z_{2}$. Since $z \in N_{1}=N_{2}$ and $0 \leqslant z_{1} z_{2} \leqslant z^{2}$, we obtain $0 \leqslant \Delta\left(z_{1} z_{2}\right)$ $\leqslant \Delta\left(z^{2}\right)=0$, which means that $z_{1} z_{2} \in N_{1}$. Hence, $N_{1} N_{1} \subset N_{1}$.

To show that $N_{1} N_{1} \subset N_{1}$ implies $N_{1}=N_{2}$ is trivial.

LEMMA 3.7. If $x \in A$ and $\Delta(x)=0$, then $x \in N_{1}-N_{1}$.

Proof. If $x \in A$ and $\Delta(x)=0$, then we may use the fact that $A$ is directed to select an element $y \in A$ such that $0 \leqslant y$ and $x \leqslant y$. Since $y-x \geqslant$ 
0 , we have $0 \leqslant \Delta(y-x) \leqslant y-x$, from which it follows that $x \leqslant y-\Delta(y)$. Defining $z_{1}=y-\Delta(y)$ and $z_{2}=z_{1}-x$, we can easily show that $z_{1}, z_{2} \in N_{1}$ and $x=z_{1}-z_{2}$. Thus, $x \in N_{1}-N_{1}$. Note that if we put $z=z_{1}+z_{2}$, then $z \in N_{1}$ and $-z \leqslant x \leqslant z$.

4. Properties which imply that $\Delta$ is multiplicative. Although we assume here that $A$ has a diagonal projection map, the reader should note that some of the properties considered do not require the existence of $\Delta$. The most important fact is the following.

Lemma 4.1. If $N_{1}=N_{2}$, then $\Delta$ is multiplicative. (By Lemma 3.5, we only need $N_{1} \subset N_{2}$.)

Proof. Since $A$ is directed, we only need to show that if $x \geqslant 1$ and $y \geqslant 1$, then $\Delta(x y)=\Delta(x) \Delta(y)$. Since $\Delta(x) \geqslant 1$ and $\Delta(x) \in A_{1}$, we must have $0 \leqslant \Delta(x)^{-1} \leqslant 1$. If we define $x_{1}=\Delta(x)^{-1} x$, then $\Delta\left(x_{1}\right)=1$ and $1 \leqslant x_{1}$ because $\Delta(x) \leqslant x$. Similarly we may define $y_{1}=y \Delta(y)^{-1}$, so that $\Delta\left(y_{1}\right)=1 \leqslant$ $y_{1}$. Now if we put $w=x_{1}-1$ and $z=y_{1}-1$, then $w, z \in N_{1}$. Since $N_{1}=$ $N_{2}$, we have $N_{1} N_{1} \subset N_{1}$ from Lemma 3.6, which means $\Delta(w z)=0$. Since $x_{1} y_{1}=1+w+z+w z$, we get

$$
1=\Delta\left(x_{1} y_{1}\right)=\Delta(x)^{-1} \Delta(x y) \Delta(y)^{-1}
$$

by using part (2) of Theorem 3.2. Hence, $\Delta(x y)=\Delta(x) \Delta(y)$.

THEOREM 4.2. If $N_{1}-N_{1}$ is a one-sided ideal (in the sense of ring theory), then $\Delta$ is multiplicative.

Proof. If $N_{1}-N_{1}$ is a one-sided ideal, then $N_{1} N_{1} \subset N_{1}$. From Lemma 3.6 it follows that $N_{1}=N_{2}$. From Lemma 4.1 it follows that $\Delta$ is multiplicative.

The reader should note that the converse of the above theorem is true and is easily verified by routine computation (but one must use Lemma 3.7); in fact, $N_{1}-N_{1}$ is a two-sided ideal. The reader should also refer to Definition 29 on p. 109 of [4].

THEOREM 4.3. Suppose $A$ has the property: if $x, y \in A, \Delta(x) \geqslant 0$ and $\Delta(y) \geqslant 0$, then $\Delta(x y) \geqslant 0$. Then $\Delta$ is multiplicative.

Proof. For any $z \in N_{1}$ we define $x=1-z$ and $y=z$. Hence, $\Delta(x)=$ $1 \geqslant 0$ and $\Delta(y)=0$. By the above property, $\Delta(x y)=\Delta\left(z-z^{2}\right) \geqslant 0$, so that $\Delta\left(z^{2}\right) \leqslant \Delta(z)=0$. Since $\Delta\left(z^{2}\right) \geqslant 0$, we obtain $\Delta\left(z^{2}\right)=0$, which means that $z \in N_{2}$. This shows that $N_{1} \subset N_{2}$ and from Lemma 4.1 it follows that $\Delta$ is multiplicative.

The property of Theorem 4.3 holds in Examples 6 through 9 but does not hold in Example 5. 
The following property is more easily understood by referring to $\S 5$ on some false conjectures.

THEOREM 4.4. Suppose $A$ has the property: if $x \in A$, then there exists $w \in N_{2}$ such that $x^{2} \geqslant-w$. (Note that this implies that $\Delta\left(x^{2}\right) \geqslant-\Delta(w)=0$.) Then $\Delta$ is multiplicative.

Proof. We first use this property to show that $N_{1} N_{2} \subset N_{1}$. If we take $z \in N_{1}$ and $w \in N_{2}$, then $\Delta\left((n w-z)^{2}\right) \geqslant 0$ for all $n=1,2, \ldots$. Therefore, $0 \leqslant n \Delta(w z+z w) \leqslant \Delta\left(z^{2}\right)$ for all $n$, which means that $\Delta(w z)=\Delta(z w)=0$ by using the Archimedean property. Hence, $N_{1} N_{2} \subset N_{1}$ and $N_{2} N_{1} \subset N_{1}$.

If we take $z \in N_{1}$, then for each positive integer $n$ there exists $w_{n} \in N_{2}$ such that $(n 1-z)^{2} \geqslant-w_{n}$. By rewriting and multiplying by $z$ on the left, we obtain $0 \leqslant 2 n z^{2} \leqslant n^{2} z+z w_{n}+z^{3}$. Since $z w_{n} \in N_{1} N_{2} \subset N_{1}$, we have $\Delta\left(z w_{n}\right)$ $=0$. Therefore, $0 \leqslant n \Delta\left(z^{2}\right) \leqslant \Delta\left(z^{3}\right)$ for all $n=1,2, \ldots$. From the Archimedean property we obtain $\Delta\left(z^{2}\right)=0$. Thus, $N_{1} \subset N_{2}$ and from Lemma 4.1 it follows that $\Delta$ is multiplicative.

The above property holds in Examples 6 through 9 but not in Example 5.

Lemma 4.5. If $x, y \in A$ are elements such that $x \leqslant 1,0 \leqslant y$ and $1 \leqslant x y$, then $0 \leqslant(1-x)^{n} \leqslant y$ for all $n=1,2, \ldots$.

Proof. See Proposition 3 on p. 640 of [3].

THEOREM 4.6. Suppose $A$ has the property: if $z \in A$ and $z \geqslant 0$, then there exists $a \in A_{1}$ such that $a \leqslant 1+z, a-z$ has an inverse and $0 \leqslant(a-z)^{-1}$. Then $\Delta$ is multiplicative.

PROof. If we take any $z \in N_{1}$, then $z \geqslant 0$, so that by the above property there exists $a \in A_{1}$ such that $a \leqslant 1+z$ and $0 \leqslant(a-z)^{-1}$. Since $a \in A_{1}$, we have $a=\Delta^{\prime}(a) \leqslant \Delta(1+z) \leqslant 1$. Therefore, $a-z \leqslant 1-z \leqslant 1$ and $1 \leqslant$ $(1-z)(a-z)^{-1}$. Taking $1-z=x$ in Lemma 4.5 , we see that $0 \leqslant z^{n} \leqslant$ $(a-z)^{-1}$ for all $n=1,2, \ldots$. From Lemma 3.4 it follows that $0 \leqslant \Delta\left(z^{2}\right) \leqslant 1$.

If $z \in N_{1}$, then $n z \in N_{1}$ for all $n=1,2, \ldots$. From the previous paragraph it follows that $0 \leqslant n^{2} \Delta\left(z^{2}\right) \leqslant 1$ for all $n=1,2, \ldots$. From the Archimedean property we obtain $\Delta\left(z^{2}\right)=0$. Thus, $N_{1} \subset N_{2}$ and from Lemma 4.1 it follows that $\Delta$ is multiplicative.

The above property holds in Examples 6 and 9 but not in Examples 5 and 7. This property is what is needed to prove an abstract version of the statement that a triangular matrix has an inverse if and only if its diagonal has an inverse.

THEOREM 4.7. Suppose $A$ has the property described in Theorem 4.6. If $x \in A$ and $x$ has a left or right inverse, then $\Delta(x)$ has a full inverse. If $\Delta(x)$ has a left or right inverse, then $x$ has a full inverse. Hence, it follows that if $x$ 
has a left or right inverse, then it has a full inverse.

Proof. Suppose $x, y \in A$ and $x y=1$. Since $\Delta$ is multiplicative, we have $\Delta(x) \Delta(y)=1$. Since $A_{1}$ is commutative, we also have $\Delta(y) \Delta(x)=1$, which means that $\Delta(y)=\Delta(x)^{-1}$.

Next suppose that $b, x \in A$ and $\Delta(x) b=1$. From part (5) of Lemma 3.1 it follows that $b \in A_{1}$ and $b \Delta(x)=\Delta(b x)=1$ (we used part (2) of Theorem 3.2 here). If we define $w=1-b x$, then $\Delta(w)=0$. From Lemma 3.7 there exists $z \in N_{1}$ such that $-z \leqslant w \leqslant z$. We now use the proof of Theorem 4.6 and Propositions 2, 3 and 4 of [3] to assert that $1-w$ has an inverse. From above we see that $x=\Delta(x)(1-w)$, which means that $x^{-1}=(1-w)^{-1} b$.

THEOREM 4.8. Suppose $A$ has the property: if $z \in N_{1}$, then there exists $a \in A_{1}$ such that $z+a z \leqslant z a$. Then $\Delta$ is multiplicative.

Proof. If $z \in N_{1}$ and $a \in A_{1}$ is as described in the theorem, then $z^{2}+$ $z a z \leqslant z^{2} a$ and $z^{2}+a z^{2} \leqslant z a z$. Hence, $2 z^{2}+a z^{2} \leqslant z^{2} a$, so that $2 \Delta\left(z^{2}\right)+$ $a \Delta\left(z^{2}\right) \leqslant \Delta\left(z^{2}\right) a$ or $\Delta\left(z^{2}\right) \leqslant 0$ (we used part (2) of Theorem 3.2 here). Therefore, $\Delta\left(z^{2}\right)=0$ so that $z \in N_{2}$. Since $N_{1} \subset N_{2}$, it follows from Lemma 4.1 that $\Delta$ is multiplicative.

This rather curious property was mentioned in Example 6 and it also holds in Example 7; in both cases upper triangular matrices are involved. It does not hold in Example 9 because the matrices are lower triangular. It also does not hold in Example 8 because the diagonal is a bounded sequence of real numbers.

THEOREM 4.9. Suppose $A$ has the property: if $x, y \in A, x \geqslant 0, y \geqslant 0$ and $x y \geqslant 1$, then there exist $a, b \in A$ such that $0 \leqslant a \leqslant x, 0 \leqslant b \leqslant y$ and $a b$ $=1$. Then $\Delta$ is multiplicative.

Proof. If $z \in N_{1}$, we define $c=\left[1+\Delta\left(z^{2}\right)\right]^{-1}$; thus, $0 \leqslant c \leqslant 1$. We next define $x=c(1+z)$ and $y=1+z$. It is easy to show that $x y \geqslant \Delta(x y)=$ 1 ; the reader should note here that $\Delta(x)=\Delta(c)=c$. Let $a, b \in A$ be as described in the theorem. Since $0 \leqslant a \leqslant x, b \leqslant y$ and $1 \leqslant y$, we obtain $x y-1$ $=x y-a b \geqslant(x-a) y \geqslant x-a \geqslant 0$ and $x y-1=x y-a b \geqslant a(y-b) \geqslant 0$. Since $\Delta(x y-1)=0$, it follows that $\Delta(a)=\Delta(x)=c$ and $\Delta(a) \Delta(y-b)=0$. Since $\Delta(a)=c$ and $c^{-1}$ exists, it follows that $1=\Delta(y)=\Delta(b) \leqslant b$. Since $a$ $\geqslant 0, b \geqslant 1$ and $a b=1$, we may use part (4) of Lemma 3.1 to assert that $b \in$ $A_{1}$, which means that $\Delta(b)=b$. Since $\Delta(b)=1$, we get $a=b=c=1$, which means that $\Delta\left(z^{2}\right)=0$. Hence, $z \in N_{2}$. Since $N_{1} \subset N_{2}$, it follows from Lemma 4.1 that $\Delta$ is multiplicative.

The above property holds in Examples 6 through 9 but not in Example 5 . 
5. Some false conjectures. By looking at triangular matrices, one might conjecture that each of the following properties implies that $\Delta$ is multiplicative.

Property 5.1. If $x, y \in A$, then $\Delta(x y)=\Delta(y x)$.

Property 5.2. If $x \in A$, then $\Delta\left(x^{2}\right) \geqslant 0$.

Property 5.3. If $x \in A$, then $x^{2} \geqslant-w$, where $w \in N_{1}$. (Compare with Theorem 4.4.)

These properties hold in Example 5, but $\Delta$ is not multiplicative in that example.

\section{REFERENCES}

1. G. Birkhoff, Lattice theory, 3rd ed., Amer. Math. Soc. Colloq. Publ., vol. 25, Amer. Math. Soc., Providence, R. I., 1967. MR 37 \#2638.

2. T.-Y. Dai, On some special classes of partially ordered linear algebras, J. Math. Anal. Appl. 40 (1972), 649-682. MR 47 \#4890.

3. R. E. DeMarr, On partially ordering operator algebras, Canad. J. Math. 19 (1967), 636-643. MR 35 \#3450.

4. A. Hopenwasser, Completely isometric maps and triangular operator algebras, Proc. London Math. Soc. (3) 25 (1972), 96-114. MR 48 \#883.

5. R. V. Kadison and I. M. Singer, Extensions of pure states, Amer. J. Math. 81 (1959), 383-400. MR 23 \#A1 243.

6. - Triangular operator algebras. Fundamentals and hyperreducible theory, Amer. J. Math. 82 (1960), 227-259. MR 22 \#12409.

7. J. von Neumann, On rings of operators. III, Ann. of Math. 41 (1940), 94-161. MR 1, 146.

8. A. L. Peressini, Ordered topological vector spaces, Harper and Row, New York, 1967. MR 37 \#3315. 1974.

9. H. H. Schafer, Banach lattices and positive operators, Springer-Verlag, Berlin,

10. B. Z. Vulikh, Introduction to the theory of partially ordered spaces, WoltersNoordhoff, Groningen, 1967. MR 37 \#121.

DEPARTMENT OF MATHEMATICS, YORK COLLEGE, CITY UNIVERSITY OF NEW YORK, JAMAICA, NEW YORK 11451

DEPARTMENT OF MATHEMATICS, UNIVERSITY OF NEW MEXICO, ALBUQUERQUE, NEW MEXICO 87131 